Research Paper

\title{
Clinical efficacy of three types of autogenous bone grafts in treatment of single-segment thoracic tuberculosis: A retrospective cohort study
}

\author{
Ke Tang\#, Jianxiao Li\#, Tianji Huang, Weiyang Zhong ${ }^{\bowtie}$, Xiaoji Luo, Zhengxue Quan ${ }^{\varpi}$ \\ Department of Orthopedic surgery, The First Affiliated Hospital of Chongqing Medical University, Chongqing, China. \\ \#Equal contributions as co-first authors. \\ $\square$ Corresponding authors: Dr Weiyang Zhong, Department of Orthopedic Surgery, The First Affiliated Hospital of Chongqing Medical University (E-mail: \\ zhongweiyang@hospital.cqmu.edu.cn); Zhengxue Quan, Department of Orthopedic Surgery, The First Affiliated Hospital of Chongqing Medical University \\ (E-mail: quanzx18@126.com).
}

() The author(s). This is an open access article distributed under the terms of the Creative Commons Attribution License (https://creativecommons.org/licenses/by/4.0/). See http://ivyspring.com/terms for full terms and conditions.

Received: 2020.04.22; Accepted: 2020.09.27; Published: 2020.10.16

\begin{abstract}
A retrospective study investigated and compared the results of lamina with spinous process (LSP), transverse process strut (TPS) and iliac graft (IG) as bone graft in thoracic single-segment spinal tuberculosis(TB) with the one-stage posterior approach of debridement, fusion and internal instrumentation. 99 patients treated from January 2012 to December 2015 were reviewed. LSP was performed in 35 patients (group A), TPS was undertaken in 33 patients (group B), and IG was carried out in 31 patients (group C). Surgical time, blood loss, hospitalization time, drainage volume, and follow-up (FU) duration were recorded. The visual analog scale (VAS), Oswestry Disability Index (ODI), erythrocyte sedimentation rate (ESR), C-reactive protein (CRP), American Spinal Injury Association (ASIA) grade, segmental angle, intervertebral height and bone fusion time were compared between preoperative and final FU. All the patients were followed up for a mean $43.90 \pm 10.39$ months in group $A$, $45.30 \pm 6.20$ months in group $B, 44.32 \pm 7.17$ months in group $C$ without difference $(P>0.05)$. The mean age was younger, the blood loss was less, the hospitalization time and the surgical time were shorter in group $A$ than those in group $B$ and $C(P<0.05)$. The drainage volume was less in group $A$ than that in group $B$ and group $C$. The CRP, ESR, VAS, and ODI were significantly decreased and there were no significant difference among the groups at the final FU. The neurological function after surgery was improved compared with preoperation among the groups. The bony fusion at a mean time $12.90 \pm 3.91$ months in group $A$ was longer than that in group $B(6.75 \pm 1.55$ months) and group $C(5.52 \pm 1.64$ months $)(P<0.05)$. No significant difference was found at the mean segmental angle, mean intervetebral height of preoperation and final FU among the groups $(P>0.05)$. In conclusion, the LSP and TPS as bone graft are reliable, safe, and effective for single-segment stability reconstruction for surgical management of thoracic TB and TPS could be new bone graft methods.
\end{abstract}

Key words: bone graft; thoracic spine; spinous process; lamina; transverse process strut; tuberculosis

\section{Introduction}

The spine tuberculosis (TB) is high risky, resulting in bone destruction, spinal deformity, and/or even paraplegia which should be paid more attention. Usually, anti-TB drugs, surgery and supportive managements are considered as effective treatment for spinal TB [1,2]. When the patients are indicated for surgery, the radical lesion debridement, solid fusion of implants and standard course of
anti-TB drugs are considered successful treatment of spine tuberculosis. Among the surgical management, the bone grafts play the key roles in curing spinal TB which could rebuild spinal stability and maintain the alignment [3-5]. Currently, the autologous iliac crest, allogeneic bone graft, rib graft, fibula and titanium mesh cages are reported widely, which are associated with longer surgery time, massive surgical blood loss 
or cages subsidence, while transverse process and lamina with spinous process(LSP) as bone grafts have rarely been reported.

To achieve the sufficient support strength and a satisfactory fusion rate, we reported two new bone grafting methods to minimize donor site complications through a posterior-only approach debridement and internal instrumentation in treatment of thoracic single-segment spinal $\mathrm{TB}$, comparing the clinical efficacy with iliac crest, to let patients recover quickly after operation.

\section{Materials and Methods}

\section{Patient selection}

The Institutional Review Board of our hospital approved this study and conducted in accordance with the Declaration of Helsinki. All participants provided written informed consent before their data were stored in our hospital database and used for study purposes. From January 2012 to December 2015, in the spine unit of the department, a total of 99 patients with thoracic single-segment spinal TB were reviewed retrospectively and weredivided into three groups. When communicating with patients before surgery, the advantages and disadvantages of the three types of bone grafts managements were fully introduced, so the patients could choose the right treatment for themselves. The surgery procedure was performed by the same spine team. The inclusion criteria were as follows: adult thoracic single-segment spinal $\mathrm{TB}$, one-stage posterior approach, internal instrumentation and reconstruction, and the patients were indicated for surgery: increasing kyphosis, neurological deficits, bone destruction affecting the stability. The exclusion criteria were as follows: active pulmonary $\mathrm{TB}$, extrapulmonary $\mathrm{TB}$, trauma, spine tumor, and spinal metastasis.

\section{Preoperative management}

Anti-TB Chemotherapy was administered as soon as the diagnosis was suggested clinically. Anti-TB drugs with the HREZ standard regimen that consists of isoniazid $(5-10 \mathrm{mg} / \mathrm{kg} /$ day), rifampicin $(10 \mathrm{mg} / \mathrm{kg} /$ day), ethambutol $(15 \mathrm{mg} / \mathrm{kg} /$ day $)$, and pyrazinamide $(25 \mathrm{mg} / \mathrm{kg} /$ day) was administered 3-4 weeks before the surgery $[2,10]$. Surgical treatment was required when the erythrocyte sedimentation rate (ESR), C-reactive protein (CRP), decreased obviously to normal, or near-normal.

\section{Surgical procedure}

After the administration of general anesthesia, the patients were placed in the prone position. The posterior spinal elements including the lamina, facet joints, and transverse processes were exposed through a midline incision. The pedicle screws of four-level or five-level were fixed based on imaging and C-arm X-ray findings, which were used to ensure its accuracy. The entire LSP or TPS was cut off using a sharpened drill or ultrasonic osteotome, and then one of the facet joints was removed for decompression and radical debridement. The LSP or TPS was trimmed by a bone knife, a sharpened drill, or an ultrasonic osteotome to obtain a suitable and threecortical-sided bone graft. Decompression and radical debridement were performed. The LSP, TPS or IG were cut off and the area was trimmed to a suitable bone graft. According to the space remaining after radical debridement, one was implanted and appropriately forced with instrumentation locking. Streptomycin $1.0 \mathrm{~g}$ and isoniazid $0.2 \mathrm{~g}$ mixed with gelatin were used locally. Negative pressure drainage was managed postoperatively, and the specimens were sent for bacterial culture and pathology.

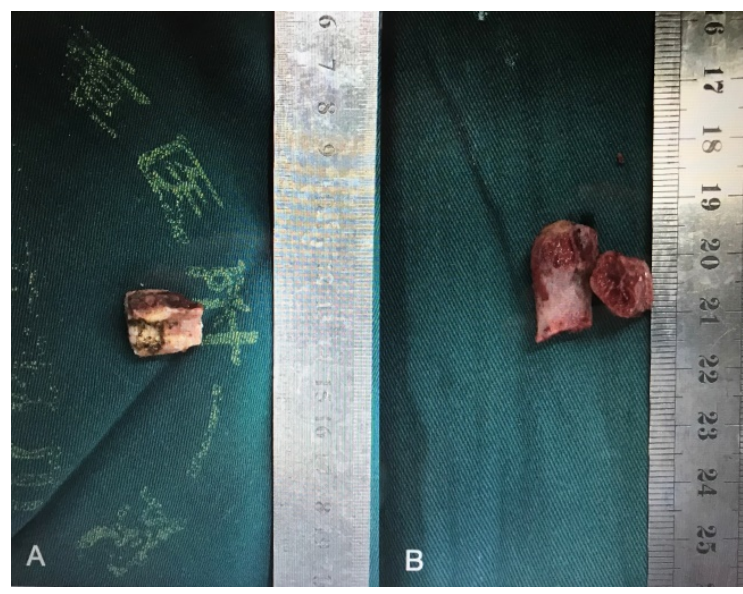

Figure 1. Photographs of trimmed LSP (A) and TPS (B).

\section{Postoperative care}

The patients continued the oral HREZ chemotherapy postoperatively. One year later, the oral pyrazinamide was stopped and the patients continued 6-month regimens of HRE chemotherapy (12HREZ/12-18HRE). Rehabilitation therapist-guided ambulation exercise was started at 1 week after operation. All patients were evaluated clinically and radiologically at 1 week, 3 months, 6 months, and 12 months after the operation and annually thereafter.

\section{Follow-up index}

For all patients, the data were observed perioperatively and during follow-up (FU): (1) the operation time, surgical blood loss, hospitalization time, drainage, FU time and bone fusion time. (2) Segmental angle. According to the Cobb method, the segmental angle was defined as the angle formed between the superior endplate of the upper vertebral 
body and the inferior endplate of the lower vertebral body. (3) Intervertebral height is defined as the vertical height between the upper and lower vertebral bodies of the fused segment on the lateral X-ray. (4) Neurological function assessed by American Spinal Injury Association (ASIA) grade. (5) VAS and ODI (6) ESR and CRP. The bone fusion was assessed using criteria of Bridwell et al. [6-7] with the X -ray and CT while necessary. All radiographic data and measurements in this study were reviewed by one senior spine surgeon and one senior radiologist.

\section{Statistical analysis}

The statistical analysis was performed using Statistic Analysis System (SAS Institute Inc., Cary, $\mathrm{NC}$, USA). The results are expressed as mean \pm SD. Differences with $P$ values $<0.05$ were considered statistically significant.

\section{Results}

\section{Clinical assessments}

All the patients were followed up for a mean

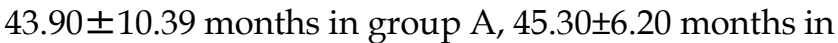
group $B, 44.32 \pm 7.17$ months in group $C$ without difference $(P>0.05)$. The mean age was younger, the blood loss was less, the hospitalization time and the surgical time were shorter in group A than those in group $\mathrm{B}$ and $\mathrm{C}(P<0.05)$. The drainage volume was less in group $A$ than that in group $B$ and group $C$ (Table 1). The CRP, ESR, VAS, and ODI were significantly decreased there were no significant difference among the groups at the final FU except the ESR was higher in group A than that in group B and group C (Table 2). The neurological function after surgery was improved compared with preoperation. In the group $A, 2$ patient improved from grade $B$ to grade $D, 5$ patients from grade $C$ to grade $D, 8$ patiens from grade $\mathrm{D}$ to grade $\mathrm{E}$. In group $\mathrm{B}, 15$ patients improved from grade $C$ to grade $D$ and 5 patients from grade $\mathrm{D}$ to grade $\mathrm{E}$. In group C, 10 patients improved from grade $C$ to grade $D$ and 3 patients from grade $\mathrm{D}$ to grade $\mathrm{E}$. There was no significant differences in ASIA grades before surgery and at final FU among the three groups $(P>0.05)$.

\section{Radiological assessments}

The thoracic spinal TB was well cured and the bony fusion at a mean time $12.90 \pm 3.91$ months in group A was longer than that in group B $(6.75 \pm 1.55$ months) and group $C(5.52 \pm 1.64$ months $)(P<0.05)$ (Figures $2 \& 3$ ). No significant difference was found at the mean segmental angle, mean intervetebral height of preoperation and final $\mathrm{FU}$ among the groups $(P>0.05)$.

Table 1. Information of patients

\begin{tabular}{|c|c|c|c|c|c|c|}
\hline & Group A & Group B & Group C & $\mathrm{P}_{\mathrm{AB}}$ & $\mathrm{P}_{\mathrm{AC}}$ & $P_{B C}$ \\
\hline No. of patients (n) & 35 & 33 & 31 & & & \\
\hline Male/female (n) & $19 / 16$ & $21 / 12$ & $15 / 16$ & & & \\
\hline Mean age (years) & $33.65 \pm 11.06$ & $47.13 \pm 18.10$ & $41.48 \pm 13.99$ & $<0.0001$ & $<0.0001$ & 0.7619 \\
\hline Hospital stay (days) & $14.05 \pm 3.58$ & $17.61 \pm 5.03$ & $17.88 \pm 10.06$ & $<0.0001$ & $<0.0001$ & 0.9168 \\
\hline Surgery time (minutes) & $182.40 \pm 23.82$ & $205.10 \pm 25.60$ & $231.40 \pm 80.90$ & 0.6327 & $<0.0001$ & $<0.0001$ \\
\hline Blood loss (ml) & $280.80 \pm 76.82$ & $301.50 \pm 165.40$ & $510.00 \pm 300.00$ & 0.8305 & $<0.0001$ & $<0.0001$ \\
\hline Drainage (ml) & $340.00 \pm 167.20$ & $450.55 \pm 171.80$ & $620.90 \pm 150.65$ & 0.0176 & 0.0192 & 0.0444 \\
\hline Mean fusion time (months) & $12.90 \pm 3.91$ & $6.75 \pm 1.55$ & $5.52 \pm 1.64$ & $<0.0001$ & $<0.0001$ & 0.3465 \\
\hline Follow-up (months) & $43.90 \pm 10.39$ & $45.30 \pm 6.20$ & $44.32 \pm 7.17$ & 0.1530 & 0.1760 & 0.9529 \\
\hline
\end{tabular}

Table 2. Clinical and radiographic outcomes

\begin{tabular}{|c|c|c|c|c|c|c|}
\hline Parameter & Group A & Group B & Group C & $\mathrm{P}_{\mathrm{AB}}$ & $\mathrm{P}_{\mathrm{AC}}$ & $\mathrm{P}_{\mathrm{BC}}$ \\
\hline \multicolumn{7}{|l|}{ ESR } \\
\hline before treatment & $79.75 \pm 7.55$ & $62.51 \pm 17.45$ & $63.72 \pm 15.61$ & 0.0126 & 0.0147 & 0.8867 \\
\hline Final FU & $13.95 \pm 4.50$ & $10.61 \pm 2.96$ & $13.36 \pm 3.13$ & 0.3044 & 0.9896 & 0.4096 \\
\hline \multicolumn{7}{|l|}{ VAS } \\
\hline before treatment & $6.95 \pm 0.94$ & $6.5 \pm 0.75$ & $6.68 \pm 1.25$ & 0.8702 & 0.7670 & 0.8023 \\
\hline Final FU & $1.95 \pm 0.69$ & $1.58 \pm 0.95$ & $1.68 \pm 0.76$ & 0.8296 & 0.8888 & 0.9607 \\
\hline \multicolumn{7}{|l|}{ ODI } \\
\hline before treatment & $39.95 \pm 4.84$ & $40.25 \pm 4.12$ & $40.76 \pm 4.74$ & 0.7247 & 0.7019 & 0.9400 \\
\hline Final FU & $4.50 \pm 1.54$ & $4.56 \pm 1.33$ & $4.88 \pm 1.11$ & 0.9417 & 0.9296 & 0.7670 \\
\hline \multicolumn{7}{|c|}{ Segmental angle $\left({ }^{\circ}\right)$} \\
\hline before treatment & $17.97 \pm 2.80$ & $18.77 \pm 2.49$ & $24.61 \pm 14.96$ & 0.6452 & 0.1638 & 0.5004 \\
\hline Final FU & $16.25 \pm 3.64$ & $15.01 \pm 3.55$ & $17.62 \pm 5.38$ & 0.7643 & 0.8090 & 0.7289 \\
\hline \multicolumn{7}{|c|}{ Intervertebral height $(\mathrm{cm})$} \\
\hline before treatment & $11.30 \pm 1.80$ & $11.70 \pm 1.90$ & $12.19 \pm 1.92$ & 0.1377 & 0.1377 & 0.1962 \\
\hline Final FU & $10.30 \pm 1.25$ & $10.60 \pm 1.50$ & $11.99 \pm 1.17$ & 0.3329 & 0.1639 & 0.9010 \\
\hline
\end{tabular}

ODI: Oswestry Disability Index; VAS: visual analog scale; ESR: erythrocyte sedimentation rate; CRP: C-reactive protein; FU: follow-up. 


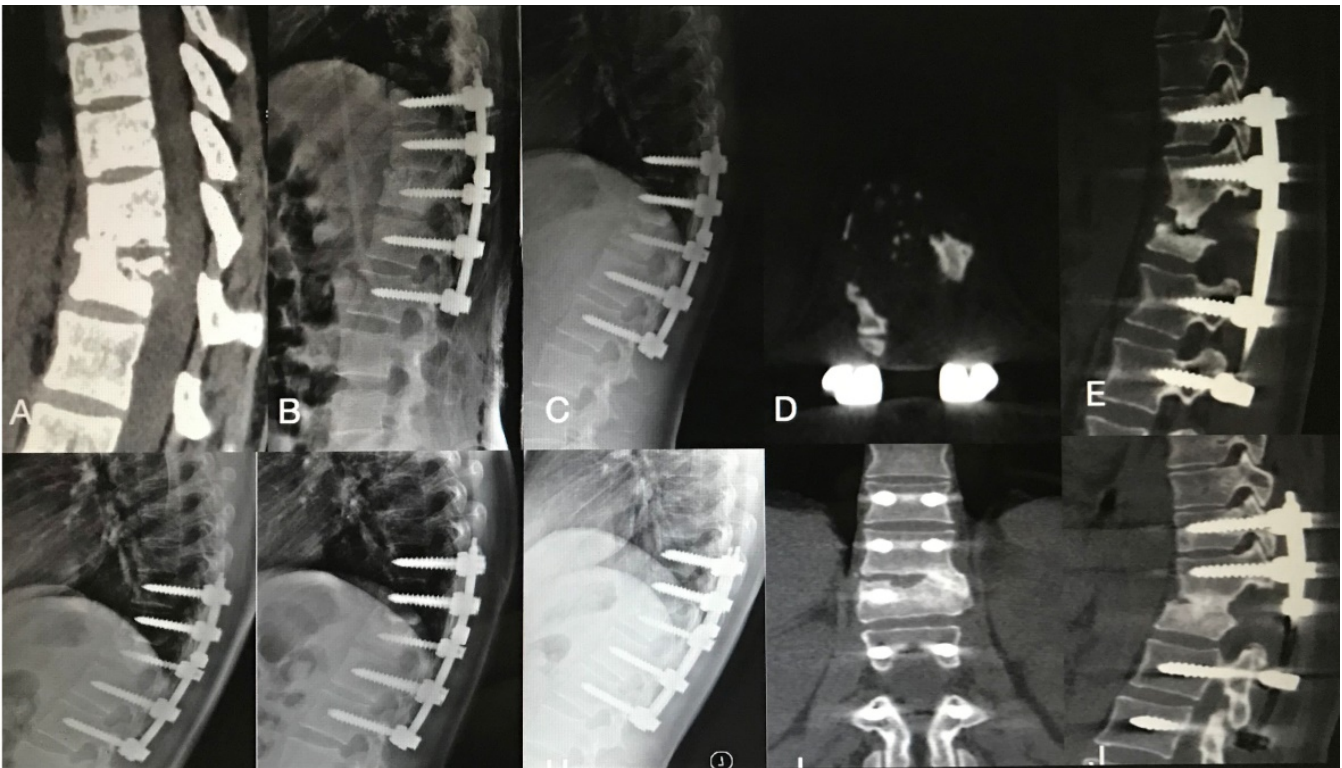

Figure 2. An 22-year-old female patient with thoracic spinal tuberculosis (T11-12) underwent posterior debridement and decompression combined with instrumentation. (A) Preoperative computed tomography (CT) showing bone destruction of the T11-12 vertebrae and compression of the spinal cord. (B,C,D,E) 1-week and 6-month postoperative $\mathrm{X}$-rays and 6-month postoperative CT showing maintained correction and the bone wasn't fully healing. (F,G) 1-year and 2-year postoperative X-rays showed kyphosis correction have loss. (H,I,J)At 4.5-year follow-up, plain X-ray, and CT show solid bone fusion.

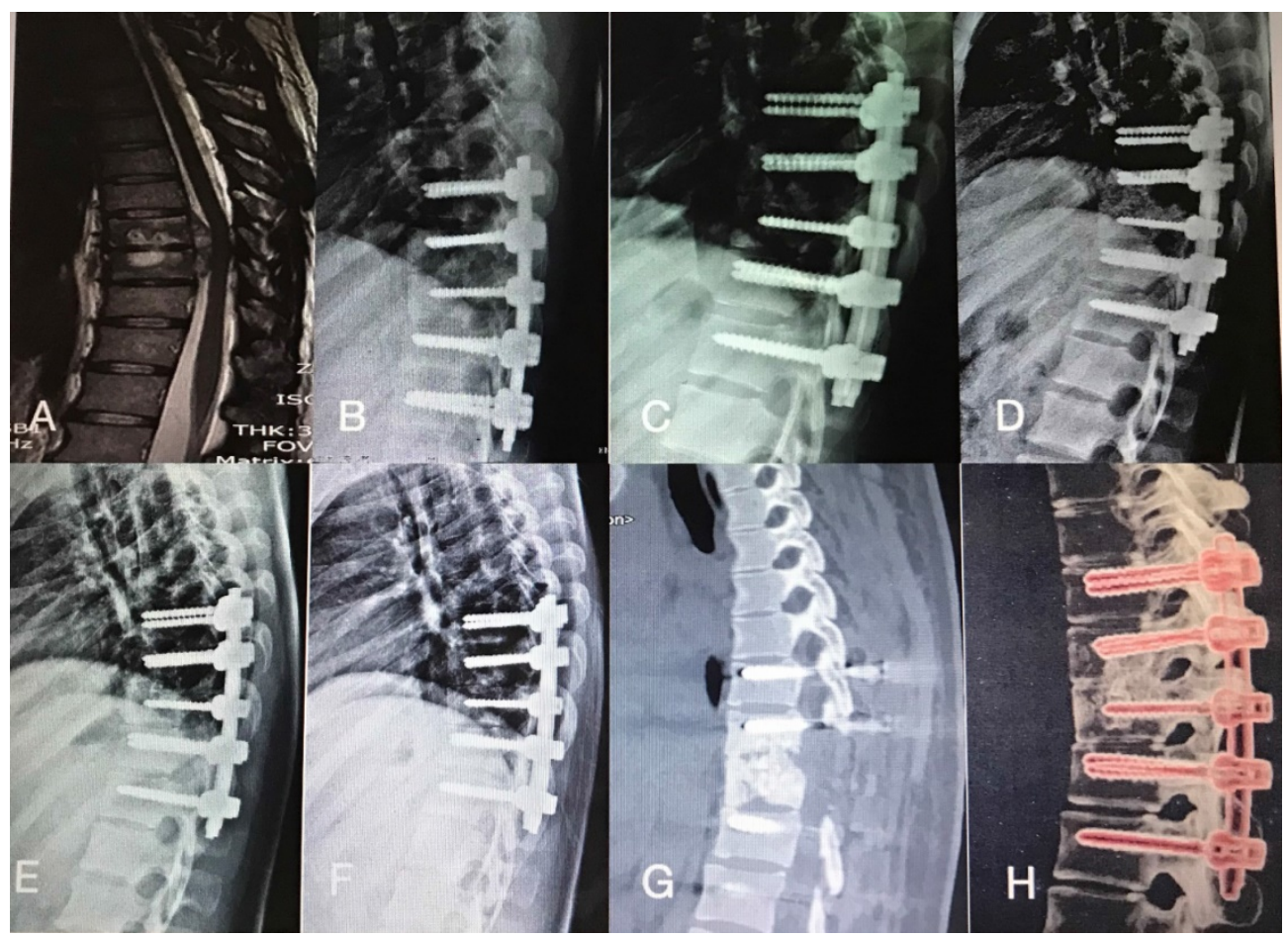

Figure 3. A 45-year-old man with thoracic spinal tuberculosis (T9-10)underwent single-segment posterior debridement and decompression combined with internal fixation. (A)Pretreatment MRI showing the destruction of the T9-10vertebrae and concomitant compression of the spinal cord. (B,C,D,E) Immediate, 3-month, 6-month, 1-year, 2-year postoperative X-ray demonstrated good location of bone graft and internal fixation. (F,G,H) At the 36-month follow-up, plain X-ray and CT showed maintenance of the correction and solid fusion.

\section{Complications}

Some postoperative complications

occurred, such as water electrolyte imbalance (one cases in group $\mathrm{A}$ and three case in group B), superficial infection of iliac donor sites (two cases in group C) which were healed by dressing changes and oral antibiotics.

\section{Discussion}

TB is a common extrapulmonary form of an ancient disease and according to the World Health Organization (WHO), TB causes 1.81 million deaths in Asia and China reports $78 \%$ new cases annually [1-3]. Human immunodeficiency virus (HIV) coinfection, bacterial resistance, childhood tuberculosis and 
population migration have caused the resurgence of all forms of TB [4-5]. Spinal TB is the most common and severe form of osteoarticular TB. Usually, formal adequate and long-term anti-TB drugs, surgery and supportive managements are considered as effective treatment for patients affected by bone destruction, sequestration, a paraspinal or spine canal abscess, or nerve impairment. Surgery via a posterior pedicle screw system with bone grafting and correction of the kyphotic deformity has been widely used for treating thoracic or lumbar spinal TB [8-11].

After complete lesion debridement, many interbody bone grafts are used to restore anterior and middle column stability. Titanium mesh cages filled with autograft have been extensively used and have high bone fusion rates. However, the problems of subsidence, stress shielding, and radio-opacity affect surgical plans [12-13]. Thus, the current study aimed to identify new bone grafting method that can provide strut support and bone fusion to decrease the complication rate [14-15].

The use of LSP and TPS as the bone graft has the following advantages. First, compared with the iliac graft, the LSP and TPS could reduce trauma and bleeding; shorten surgical, decrease postoperative drainage volumes, and postoperative complication rates. The VAS and ODI were improved significantly at final FU. Second, in our study, all patients achieved the bony fusion at a mean time 12.90 \pm 3.91 months in group $\mathrm{A}$ was longer than that in group $\mathrm{B}(6.75 \pm 1.55$ months) and group $\mathrm{C}(5.52 \pm 1.64$ months $)$. Although the LSP gained longer time of bone healing, no significance was found between the TPS and IG. At the correction of segmental kyphosis, there was no significance among the groups. Hence, the LSP and TPS could provide good support, strength, and fusion properties, and the bony fusion time of TPS was nearly the same with IG. Furthermore, LSP and TPS, as an autogenous bone, have a cortical bone structure supporting bone defect space, and could ensure and maintain the segmental stability and alignment. And comparing with LSP, the TPS could achieve shorter bony fusion time and good clinical results, which could be recommended as bone graft for treatment of single-level thoracic TB [16-17].

However, we declare that the retrospective nature of the small-sample study may be associated with bias. Second, single LSP as bone graft had long bony fusion time and may be risk for delay of the bony fusion. Third, the study didn't consider the intra- and inter-observer differences and biases. Fourth, the biomechanical testing of human cadaver spines will be verified to ensure the strong support. In the future, the prospective, randomized studies with long-term follow-up periods are needed.

\section{Conclusion}

Our study results showed that use of the LSP or TPS could be one choice in the surgical management of single-segment thoracic spinal $\mathrm{TB}$, resulting in good bone fusion and spinal stability restoration, is a reliable, safe, and effective bone grafting method. Furthermore, the TPS could be a new bone graft method.

\section{Abbreviations}

TB: tuberculosis; LSP: lamina with spinous process; TPS: transverse process strut; IG: iliac graft; ODI: Oswestry Disability Index; VAS: visual analog scale; ESR: erythrocyte sedimentation rate; CRP: C-reactive protein; FU: follow-up; ASIA: American Spinal Injury Association; HIV: human immunodeficiency virus; WHO: World Health Organization.

\section{Competing Interests}

The authors have declared that no competing interest exists.

\section{References}

1. Dunn RN, Ben Husien M. Spinal tuberculosis: review of current management. Bone Joint J. 2018;100(B4):425-431.

2. Schito M, Migliori GB, Fletcher HA, et al. Perspectives on Advances in Tuberculosis Diagnostics, Drugs, and Vaccines. Clin Infect Dis. 2015; 61(Suppl 3):102-18.

3. Kim JH, Ahn JY, Jeong SJ, et al. Prognostic factors for unfavourable outcomes of patients with spinal tuberculosis in a country with an intermediate tuberculosis burden: a multicentre cohort study. Bone Joint J. 2019;101-B(12):1542-1549.

4. Liao Y, Ye R, Tang Q, et al. Is It Necessary to Perform the Second Surgery Stage of Anterior Debridement in the Treatment of Spinal Tuberculosis? World Neurosurg. 2020;134:e956-e967.

5. Zeng Y, Cheng P, Tan J, et al. Comparison of three surgical approaches for thoracolumbar junction (T12-L1) tuberculosis: a multicentre, retrospective study. BMC Musculoskelet Disord. 2019;20(1):524.

6. Eck KR, Lenke LG, Bridwell KH, et al. Radiographic assessment of anterior titanium mesh cages. J Spinal Disord. 2000;13(6):501-510.

7. Bridwell KH, Lenke LG, McEnery KW, et al.. Anterior fresh frozen structural allografts in the thoracic and lumbar spine. Spine (Phila $\mathrm{Pa}$ 1976) $1995 ; 20: 1410-1418$.

8. Yang $\mathrm{X}$, Luo C, Liu L, et al. Minimally invasive lateral lumbar intervertebral fusion versus traditional anterior approach for localized lumbar tuberculosis: a matched-pair case control study. Spine J. 2020;20(3):426-434

9. Tang Y, Wu WJ, Yang S, et al. Surgical treatment of thoracolumbar spinal tuberculosis-a multicentre, retrospective, case-control study. J Orthop Surg Res.2019;14(1):233.

10. Sharma A, Chhabra HS, Chabra T, et al. Demographics of tuberculosis of spine and factors affecting neurological improvement in patients suffering from tuberculosis of spine: a retrospective analysis of 312 cases. Spinal Cord. 2017;55:59-63.

11. Shi T, Zhang Z, Dai F, et al.. Retrospective study of 967 patients with spinal tuberculosis. Orthopedics. 2016;39(e):838-843.

12. Shen $X$, Liu $H$, Wang $G$, et al. Single-stage posterior-only approach treating single-segment thoracic tubercular spondylitis. Int J Clin Exp Pathol. 2015;8:11051-11059.

13. Sun D, Zhang ZH, Mei G, et al. Comparison of Anterior only and Combined Anterior and Posterior Approach in Treating Lumbosacral Tuberculosis.Sci Rep. 2019;9(1):18475

14. Gao Y, Ou Y, Deng Q, et al. Comparison between titanium mesh and autogenous iliac bone graft to restore vertebral height through posterior approach for the treatment of thoracic and lumbar spinal tuberculosis. PLoS One. 2017;12(e):0175567. 
15. Pan Z, Luo J, Yu L, et al. Debridement and reconstruction improve postoperative sagittal alignment in kyphotic cervical spinal tuberculosis. Clin Orthop Relat Res. 2017;475:2084-2091.

16. Yang H, Song F, Zhang L, et al. Management of spine tuberculosis with chemotherapy and percutaneous pedicle screws in adjacent vertebrae: a retrospective study of 34 cases. Spine (Phila Pa 1976). 2017;41:E1415-E1420.

17. Sharma A, Chhabra HS, Chabra T, et al. Demographics of tuberculosis of spine and factors affecting neurological improvement in patients suffering from tuberculosis of spine: a retrospective analysis of 312 cases. Spinal Cord. 2017;55:59-63. 